Proc. Indian Acad. Sci. (Chem. Sci.), Vol. 108, No. 6, December 1996, pp. 533-538.

(C) Printed in India.

\title{
Atomic design of $\pi$-conjugated metal complex oligomers and polymers
}

\author{
HIROSHI NISHIHARA \\ PRESTO, JRDC, and Department of Chemistry, Faculty of Science and Tech- \\ nology, Keio University, 3-14-1 Hiyoshi, Kohoku-ku, Yokohama 223, Japan \\ Present address: Department of Chemistry, School of Science, The University of \\ Tokyo, 7-3-1 Hongo, Bunkyo-ku, Tokyo 113, Japan
}

\begin{abstract}
As a model to understand electronic communication between transition metal centres in $\pi$-conjugated metal complex polymers, redox properties of soluble oligo(1,1'-ferrocenylene)s were investigated. The dependence of redox potentials on the number of redox nuclei can be explained using not only the neighboring site interaction energies, $U_{\mathrm{OR}}, U_{\mathrm{OO}}$ and $U_{\mathrm{RR}}$, but also the second neighbouring site interaction energy, $U_{\text {OXR }}$. IR-spectroelectrochemistry of the (cyclopentadiene) $\mathrm{Fe}(\mathrm{CO})_{3}$-attached ferrocene, biferrocene and terferrocene has proved the theoretical prediction on the thermodynamically favorable forms of electronic isomers in the mixed valence states based on the neighboring site interaction energy.
\end{abstract}

Keywords. $\pi$-Conjugated multinuclear complexes; redox potential; oligo(1,1'-ferrocenylene)s; neighbouring site interaction energy.

\section{Introduction}

In $\pi$-conjugated multinuclear complexes, strong electronic and spin-spin interaction between metal sites through conjugated chains manifests unique electronic, optical and magnetic properties. Elongation of the conjugated chains can yield a new class of fascinating materials with various novel physical functions, that can be called "conducting metal complex polymers". In recent years, a variety of metal complex polymers belonging to this family has been extensively investigated (Nishihara 1996).

Conjugated metal complex polymers can be roughly classified into two categories by the difference in positioning of metal centers towards the conjugated chain (figure 1). Polymers in the first category, group I, involve metals in the polymer backbone such as polymetallocenylenes, polydecker complexes, and metal polyynes (Nishihara 1996). Polymers in the second one, group II, comprise organic conjugated main chain and metal sites located in pendant groups such as poly(alkynylferrocene), poly(arylene) complexes, and poly (metallacyclopentadienylene)s (Nishihara 1996). For the polymers in group I, energy bands are made of both ligand $\pi$-orbitals and metal $d$-orbitals, and band width is narrow and band gap is large when non-bonding or weakly bonding $d$-orbitals are involved. On the one hand, polymers in group II can be regarded as part of the family of so-called "conducting polymers" such as polyacetylene or polythiophene, so that band width is larger and band gap smaller than those in group I in most cases. Metal $d$-orbitals may localize and act as electron trapping sites. .

In conjugated metal complex polymers, the degree of electronic communication between metal sites dominates their electronic and magnetic properties. This paper presents two topics on such metal-metal electronic communication using oligo(1,1'ferrocenylene)s belonging to group I as examples. 
(a)

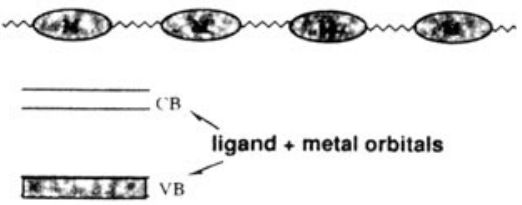

(b)

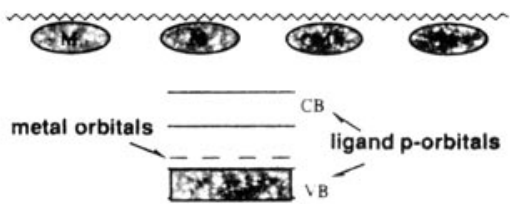

Figure 1. Classification of $\pi$-conjugated metal complex polymers involving (a) metals in the polymer backbone and (b) linearly conjugated organic chains with metals in the pendant groups (b).

\section{Redox potential dependency on the number of ferrocene units in oligo(1,1'-ferro- cenylene)}

Polymetallocenylenes (Neuse 1981) represent a class of organometallic polymers in which metallocene units are directly interconnected so as to constitute a linear chain and cause intra-chain electronic interaction between metallocene units. Poly (1,1'-ferrocenylene) is the most popular one, and its electrochemistry is an interesting subject of research to recognize how the sequence of the redox site interaction dominates the redox property of the whole system because ferrocene is a representative molecule that undergoes reversible $1 e^{-}$ oxidation and thus the polyferrocenylene system is an ideal model of a linearly combined multi-redox system.

A theoretical investigation on the electrochemical behavior of a general multi-redox system is given by Aoki and Chen (1995). This theory is constructed based on three interaction parameters between neighboring sites, $U_{\mathrm{OO}}, U_{\mathrm{RR}}$ and $U_{\mathrm{OR}}(\mathrm{O}$ and $\mathrm{R}$ refer to reduced and oxidized units respectively), and predicts that a system with an odd number of redox centers, $n=2 m+1(m \geqq 1)$ gives three redox waves involving $m, 1$ and $m$ electrons, and the one with an even number of redox sites, $n=2 m+2(m \geqq 1)$ gives four redox waves with $m, 1,1$ and $m$ electrons, and consequently, the number of waves converges into two when $m$ approaches infinity.

Electrochemical properties of unsubstituted oligoferrocenylenes have been examined up to a tetramer (Brown et al 1975), showing the same number of $1 e^{-}$oxidation waves as that of ferrocene units in a molecule due to formation of mixed valence states. Cyclic voltammetry of a low molecular weight polyferrocenylene (m.w. $\approx 900$ ), dissolved in $\mathrm{CH}_{2} \mathrm{Cl}_{2}$ and electrodeposited on $\mathrm{Pt}$, gives a broad redox wave with two (or three) peaks between 0.2 and $0.8 \mathrm{~V}$ vs SSCE (Oyama et al 1988). It should be noted that the poor solubility of unsubstituted poly( 1,1 '-ferrocenylene) is an essential problem in studying its fundamental properties.

We have recently synthesized oligo(1,1'-dihexylferrocenylene)s from dihexylfulvalene ion and $\mathrm{FeCl}_{2}(\mathrm{THF})_{2}$, and isolated oligo(dihexylferrocenylene)s up to a heptamer by the recycling GPC method (scheme 1) (Hirao et al 1996). Higher oligomers are still soluble in common organic solvents and can be used for the study to examine Aoki's theory using the neighboring site interaction. Comparison between the theoretical and experimental redox potential values for $\underline{3 a}-\underline{5 a}$ and $\underline{3 b}-\underline{6 b}$ are given in figure 2 , in which both inconsistency and consistency are recognized. Consistency is the coarse dependence of $E^{0}$ values on $n$, especially the critical difference between when $n$ is odd and when it is even, and inconsistency is the fact that theoretically undivided two-electron waves for the pentamers and the hexamer are experimentally separated into two $1 e^{-}$waves.

It has been found that the discrepancy between theoretical and experimental values as noted above can be eliminated by introducing an additional parameter, $U_{\text {OXR }}$, 
Atomic design of $\pi$-conjugated metal complex oligomers and polymers 535
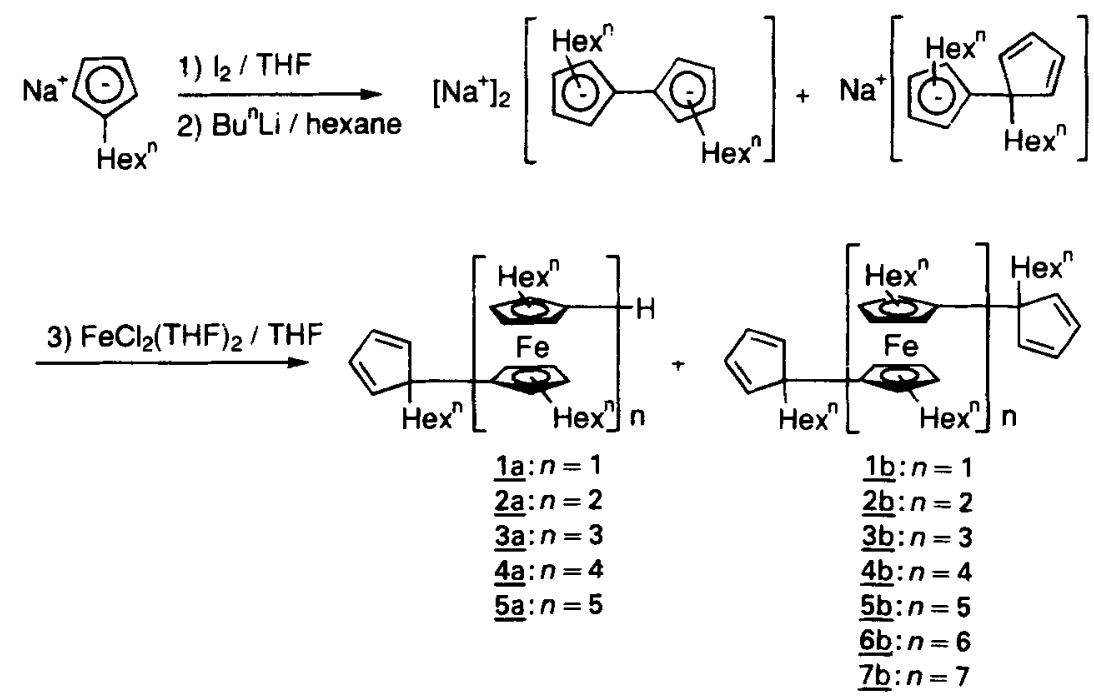

Scheme 1.

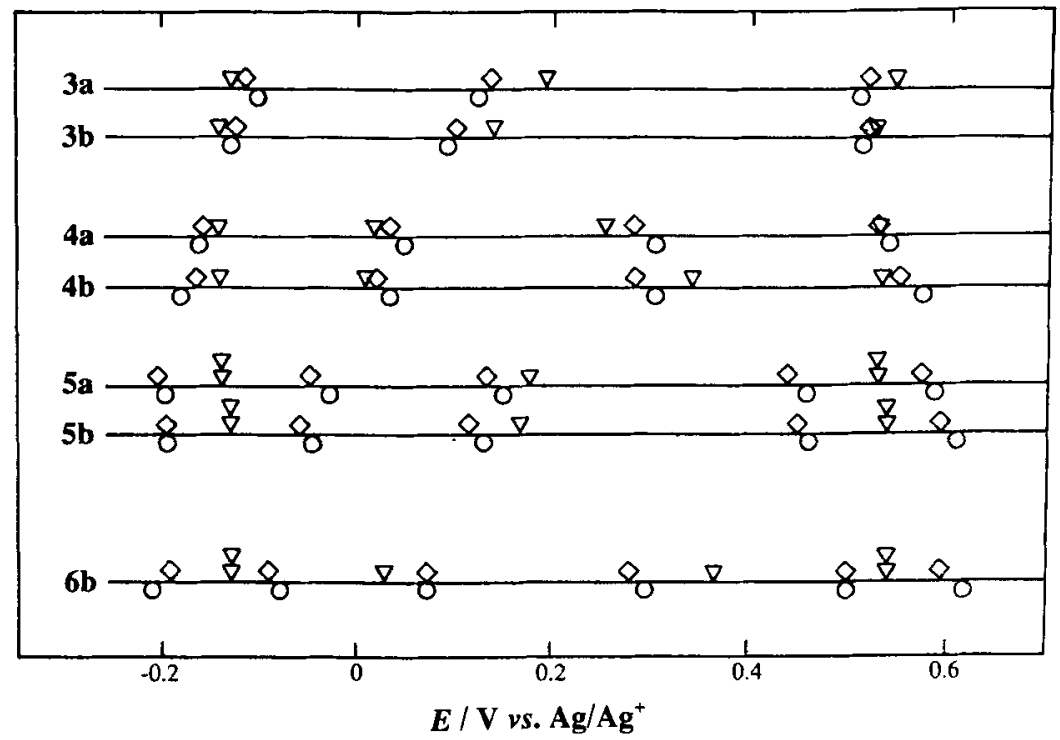

Figure 2. Formal potentials of oligo(1,1'-dihexylferrocenylene)s estimated from cyclic voltammograms $(O)$, calculated from neighboring site interaction energies $(\nabla)$ and calculated from both neighboring and second neighboring site interaction energies $(\diamond)$.

expressing second neighboring site interaction (Aoki et al 1996). This interaction causes different redox potential values between the first and the last two-electron transfer reactions for the pentamers and the hexamer as shown in figure 2. The $U_{\mathrm{OR}}$ and $U_{\text {OXR }}$ values used for this simulation are $-15 \sim-25 \mathrm{~kJ} \mathrm{~mol}^{-1}$ and $-4 \mathrm{~kJ} \mathrm{~mol}^{-1}$, respectively. We assume that the ratio $U_{\mathrm{OXR}} / U_{\mathrm{OR}}$ expresses the degree of electron 


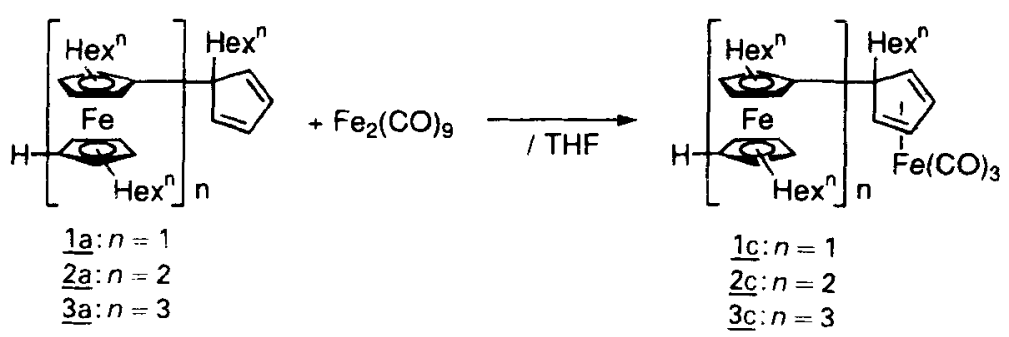

Scheme 2.

delocalization. In other words, if $U_{\mathrm{OXR}}$ is similar to $U_{\mathrm{OR}}$, the electron delocalization is very large, generating metallic conductivity. In the case of oligo(1,1'-ferrocenylene), $U_{\text {OXR }} / U_{\text {OR }}$ is small $(\approx 0 \cdot 2)$ and this may correspond to relatively low electric conductivity of poly(1,1'-ferrocenylene) doped with $\mathrm{I}_{2}$ or TCNQ (Pittman and Sasaki 1975; Sanechika et al 1981).

\section{IR-spectroelectrochemical study on the electronic structure of mixed-valence states of oligo(1,1'-ferrocenylene)}

Theory based on the neighboring site interaction noted above determines the thermodynamically most favoured electronic isomer in the mixed valence states of the multi-redox center system. For example, a redox trimer gives two possible electronic isomers in its two mixed valence states, and the theory indicates that $R-O-R$ is thermodynamically more favorable than $\mathrm{O}-\mathrm{R}-\mathrm{R}$, and also that $\mathrm{O}-\mathrm{R}-\mathrm{O}$ is more favorable than $\mathrm{O}-\mathrm{O}-\mathrm{R}$. We have attempted to recognize the electronic isomers experimentally using IRAS for ferrocene, biferrocene and terferrocene derivatives involving an iron tricarbonyl moiety.

The $\mathrm{Fe}(\mathrm{CO})_{3}$-containing ferrocenes, $\underline{1 \mathrm{c}}-\underline{3 \mathrm{c}}$, are prepared by reaction of the complexes with a free cyclopentadienyl group, $1 \mathrm{a}-\underline{3 \mathrm{a}}$, and $\mathrm{Fe}_{2}(\mathrm{CO})_{9}$ (scheme 2) (Hirao 1996). It is expected that in this structure, the infrared absorption of CO can monitor the oxidation state of the terminal ferrocenyl group as the CO stretching vibration of metal carbonyls acts as a good sensor of the electronic state of metal centers. Cyclic voltammetry of $\underline{1} \underline{c}-\underline{3 c}$ indicates that all the oxidation of ferrocene units occur at potentials more negative than that of the iron carbonyl unit, implying that the change of electronic structure of ferrocene units can be seen without oxidative decomposition of the iron cárbonyl unit.

In the IRAS spectra of the monomer, $\underline{1 \mathrm{c}}$, a wavenumber shift of the $\mathrm{CO}$ stretching mode to a higher wavenumber is observed according to the $1 e^{-}$oxidation. The shift is 12 to $15 \mathrm{~cm}^{-1}$ (see table 1) and reversible upon oxidation and reduction, indicating actually that this mode can sense the oxidation state of the ferrocene unit bound to the (cyclopentadiene) $\mathrm{Fe}(\mathrm{CO})_{3}$ unit. The possibility that the observed species is not a solute but an adsorbate and that the wavenumber shift is due to the electric field is denied by the fact that no single peak appears at intermediate wavenumbers, but owing to the reduced and oxidized forms two peaks coexist around the formal potential.

The wavenumber shifts from the fully reduced to the fully oxidized form for the dimer, $2 \mathrm{c}$, are 13 and $17 \mathrm{~cm}^{-1}$, similar to that for the monomeric complex (see table 1). In the monocationic form, the mixed valence state, the peak appears at the intermediate position between the peaks for $\underline{2 c}$ and $\underline{2 c}^{2+}$, and the peak width is much broader. This 
Table 1. $v(\mathrm{CO})$ for each oxidation state of $\underline{1 \mathrm{c}}, \underline{2 \mathrm{c}}$ and $\underline{\mathrm{zc}}$.

\begin{tabular}{|c|c|c|}
\hline \multirow{2}{*}{$\frac{\text { Compound }}{\frac{1 \mathrm{c}}{1}}$} & \multicolumn{2}{|c|}{ Wavenumber $\left(\mathrm{cm}^{-1}\right)$} \\
\hline & 2039 & 1965 \\
\hline$\underline{1 c}^{+}$ & 2051 & 1980 \\
\hline$\underline{2 c}$ & 2038 & 1965 \\
\hline$\underline{2 c}^{+}$ & 2047 & 1976 \\
\hline$\underline{2 c}^{2+}$ & 2051 & 1982 \\
\hline$\underline{3 c}$ & 2038 & 1964 \\
\hline$\underline{3 \mathrm{c}}^{+}$ & 2041 & 1970 \\
\hline $3 \mathrm{c}^{2+}$ & 2050 & 1979 \\
\hline$\underline{3 c^{3+}}$ & 2052 & 1982 \\
\hline
\end{tabular}

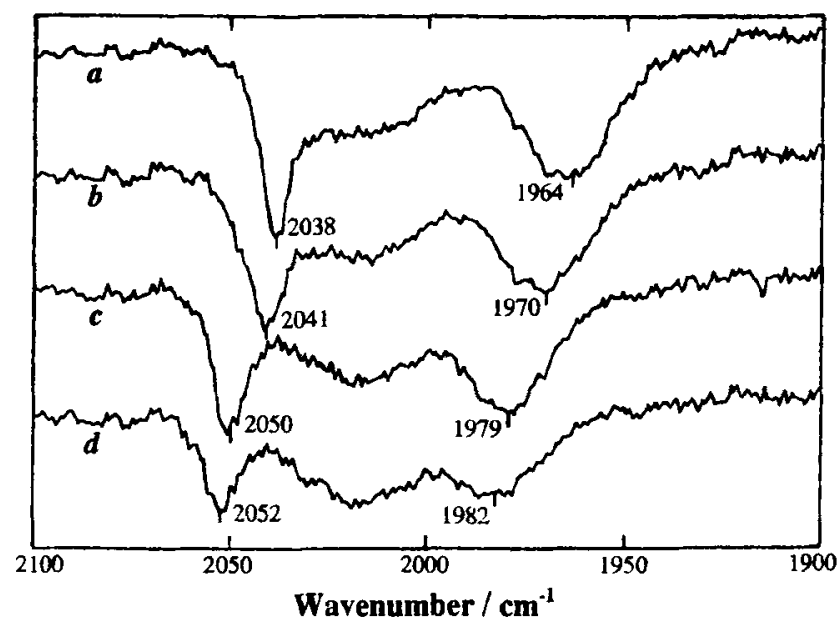

Figure 3. IRAS spectra of $3 \mathrm{c}$ when its electrolysis was carried out at a $\mathrm{Pt}$ plate in $0.1 \mathrm{~mol} \mathrm{dm}^{-3} \mathrm{Bu}_{4} \mathrm{NClO}_{4}-\mathrm{CH}_{2} \mathrm{Cl}_{2}$ at $-0.60 \mathrm{~V}(\mathrm{a}), 0 \mathrm{~V}(\mathrm{~b}), 0.35 \mathrm{~V}$ (c) and $0.65 \mathrm{~V}$ (d) vs $\mathrm{Ag} / \mathrm{Ag}^{+}$.

implies that the carbonyl group feels both the oxidized and reduced forms of the ferrocene unit neighboring the iron carbonyl moiety. This is in accordance with the expectation that the (cyclopentadiene) $\mathrm{Fe}(\mathrm{CO})_{3}$-attached ferrocene unit can be either $\mathrm{R}$ or $\mathrm{O}$ if the attached ferrocene and the non-attached ferrocene are electronically not quite different.

The IR spectra for the trimer, $\underline{3 c}$, are shown in figure 3 . In this case the consideration based on neighboring site interaction indicates that the three-step oxidation pathway is composed of $\mathrm{R}-\mathrm{R}-\mathrm{R}-\mathrm{Fe}(\mathrm{CO})_{3} \rightarrow \mathrm{R}-\mathrm{O}-\mathrm{R}-\mathrm{Fe}(\mathrm{CO})_{3} \rightarrow \mathrm{O}-\mathrm{R}-\mathrm{O}-\mathrm{Fe}(\mathrm{CO})_{3} \rightarrow \mathrm{O}-\mathrm{O}-$ $\mathrm{O}-\mathrm{Fe}(\mathrm{CO})_{3}$ and thus the (cyclopentadiene) $\mathrm{Fe}(\mathrm{CO})_{3}$-attached ferrocene site should change from $\mathrm{R}$ to $\mathrm{O}$ at the second oxidation step from monocation to dication. This prediction is roughly correct as can be seen in figure 3 that the most significant wavenumber shift is $9 \mathrm{~cm}^{-1}$ at the second step. It should be mentioned that small wavenumber shifts are observed even at the first and the third oxidation steps. It is easily evaluated that the shifts at the first and the third steps correspond to about $20 \%$ and $80 \%$ oxidation of the (cyclopentadiene) $\mathrm{Fe}(\mathrm{CO})_{3}$-attached ferrocene site if the total 
wavenumber shift ( 14 and $18 \mathrm{~cm}^{-1}$ ) corresponds to the full change in charge density of the ferrocene site from 0 to 1 . We assume that this partial charge density value can be regarded as the degree of electron delocalization.

\section{Conclusion}

The relation of redox potentials to the number of ferrocene units in penta $\left(1,1^{\prime}\right.$ ferrocenylene) and hexa(1,1'-ferrocenylene) cannot be explained by using only neighboring site interaction energies, $U_{\mathrm{OR}}, U_{\mathbf{R R}}$ and $U_{\mathrm{OO}}$, but introduction of an additional parameter, the second neighboring site interaction energy, $U_{\text {OXR }}$ can successfully simulate the experimental data. The wavenumber shifts for $v(\mathrm{CO})$ of (cyclopentadiene) $\mathrm{Fe}(\mathrm{CO})_{3}$-attached ferrocene, biferrocene and terferrocene correspond sufficiently, to the theoretically expected structures of the thermodynamically most favorable electronic isomers in the mixed valence states, based on neighboring site interaction energies. It is assumed that the ratio of $U_{\text {OXR }}$ to $U_{\text {OR }}$ in the first case and the partial charge density change estimated from the wavenumber shift $v(\mathrm{CO})$ in the second case are indicators of electron delocalization.

\section{Acknowledgements}

This work was partly supported by a Grant-in Aid for Scientific Research, No. 07215277 and 07640761 , from the Ministry of Education, Science and Culture of Japan. I thank Prof. K Aoki at Fukui University for helpful discussions on the electrochemical behaviors, and Prof. M Ito at Keio University for IRAS measurements.

\section{References}

Aoki K and Chen J 1995 J. Electroanal. Chem. 38035

Aoki K, Chen J, Nishihara H and Hirao T 1996 J. Electional. Chem. (in press)

Brown G M, Meyer T J, Cowan D O, LeVanda C, Kaufman F, Roling P V and Rausch M D 1975 Inorg. Chem. 14506

Hirao T 1996 Synthesis of novel oligo (ferrocenylene) derivatives and the relation of their physical properties to the degree of polymerization, MS thesis, Keio University, Yokohama

Hirao T, Kurashina M, Aramaki K and Nishihara H 1996 J. Chem. Soc., Dalton Trans. 2929

Neuse E W 1981 J. Macromol. Sci.-Chem. A16 3, and references therein

Nishihara H 1996 Organometallic conductive polymers. In Handbook of organic conductive molecules and polymers (ed.) H S Nalwa (Wiley) vol. 2, pp 799-832, and references therein

Oyama N, Takizawa Y, Matsuda H, Yamamoto T and Sanechika K 1988 Denki Kagaku 56781

Pittman C U Jr and Sasaki Y 1975 Chem. Lett. 383

Sanechika K, Yamamoto T and Yamamoto A 1981 Polym. J. 13255 